\title{
Prevention of Headache in Adolescents: Population-Attributable Risk Fraction for Risk Factors Amenable to Intervention
}

\author{
Lucia Albers ${ }^{1} \quad$ Astrid Milde-Busch ${ }^{1} \quad$ Otmar Bayer $^{1} \quad$ Steffi Lehmann $^{1} \quad$ Christina Riedel $^{1}$ \\ Michaela Bonfert $^{2} \quad$ Florian Heinen ${ }^{2}$ Andreas Straube ${ }^{3}$ Rüdiger von Kries ${ }^{1}$
}

${ }^{1}$ Division of Epidemiology, Institute of Social Paediatrics and Adolescent Medicine, Ludwig-Maximilians-University Munich, Munich, Germany

2 Department of Paediatric Neurology and Developmental Medicine, Hauner Children's Hospital, University of Munich, Munich, Germany ${ }^{3}$ Department of Neurology, University of Munich, Munich, Germany

\begin{abstract}
Address for correspondence and reprint requests Lucia Albers, Dipl Math, Institute of Social Paediatrics and Adolescent Medicine, LudwigMaximilians-University Munich, Heiglhofstraße 63, 81377 Munich, Germany (e-mail: lucia.albers@med.uni-muenchen.de).
\end{abstract}

\begin{abstract}
Introduction Several risk factors for headache have been identified, some of which are potentially amenable to interventions. The potential effect of such interventions can be predicted by the population-attributable risk fraction (PARF). We assessed PARFs of the the following risk factors: neck muscle pain, chronic stress, alcohol consumption, smoking, coffee consumption, and physical inactivity. We studied the maximal possible effect achievable by avoidance of these risk factors.

Methods Two approaches to estimate PARFs are compared, which assess their cumulative and individual impact of risk factors by age: the Levin formula and the average attributable fraction.
Keywords
- headache
- tension-type headache
- migraine
- risk factors
- PARF
- adolescents

\section{Introduction}

Primary headache is one of the most common health complaints among adolescents. ${ }^{1}$ The most recent review about prevalence of headache (Stovner and Andree, 2010) ${ }^{2}$ shows that the prevalence of headache in children and adolescents is on average $53 \%$ ( 29 to $77 \%$ ). Several risk factors for headache in adolescents have been identified. Some of these risk factors pertaining to stress, lifestyle, and muscular imbalances are potentially amenable to interventions. ${ }^{3-5}$

The strength of the association is the most important risk indicator for counseling the individual patient. To predict the potential impact of interventions against these risk factors on a population level, however, both the prevalence of the risk factors and the strength of the association need to be taken received

September 19, 2012 accepted

October 22, 2012

published online

January 8, 2013
Issue Theme Headaches in Childhood and Adolescence; Guest Editor, Florian Heinen, MD.
DOI http://dx.doi.org/

(C) 2013 Georg Thieme Verlag KG Stuttgart · New York 10.1055/s-0032-1332742. ISSN 0174-304X. 
into account. This is usually described as the populationattributable risk fraction (PARF), which reflects the proportion of cases that can be attributed to a certain risk factor in a population. Knowledge of the PARF of risk factors potentially amenable to preventive interventions is a prerequisite for sample size estimation in planning such studies: What is the maximum effect to be expected from removal of these risk factors in the population for whom the intervention is designed? Such knowledge about the attributable risk factors would help to focus the intervention in population-based approaches on those factors that have the highest impact on headache prevalence.

The classical method to assess the PARF is the Levin formula (Approach 1). ${ }^{6}$ This approach has a couple of limitations. It is, for example, subject to overestimation and becomes a biased estimator even when adjusted odds ratios are entered into the equation. ${ }^{7}$ Therefore, several alternatives are discussed in the literature. ${ }^{8-10}$ According to these, obtaining the PARF directly from logistic regression models as, for example, the maximum likelihood estimator of the attributable fraction introduced by Greenland and Drescher ${ }^{10}$ is more robust. Further elaboration of this approach, taking into account a sequential removal of the risk factor, is presented by Eide and Gefeller ${ }^{11}$ (Approach 2) and may thus constitute the best approach to assess PARF in cross-sectional studies. ${ }^{9}$

We used recently published data from a cross-sectional study on grammar schools in Munich, Germany, ${ }^{3-5}$ which identified lifestyle factors such as physical inactivity, smoking, coffee consumption, alcohol consumption (cocktails, alcoholic mixed drinks, liquor), ${ }^{12}$ stress $^{3}$ and muscle pain in the head, neck, and shoulder region ${ }^{5}$ as potentially modifiable risk factors for headache in adolescents. Based on these data, PARFs were estimated using different approaches to answer the following questions:

1. Which proportion of the global population risk for headache in adolescents can be explained by muscle pain in the head, neck, and shoulder region; chronic stress; alcohol consumption; smoking; coffee consumption; and physical inactivity?

2. Which risk factors are most important on a population level?

3. How does the impact of these risk factors differ between migraine and tension-type headache (TTH)?

4. Does the impact of these risk factors on a population level vary by age in adolescents?

\section{Methods}

In total 1,260 students of the 10th and 11th grade (aged between 14 and 20 years) of 11 public grammar schools in Munich, Germany, filled in a questionnaire on headache and associated risk factors. Recruitment procedures were described previously. ${ }^{3}$ Written informed consent was obtained from the parents of the participants, and the study was approved by the Bavarian Ministry for Education and Culture, the Data Safety Officer, and the Ethics Committee of the Medical Faculty of the Ludwig-Maximilians-University Munich. Consent of the participants was assumed when they handed over the completed questionnaires to the study members.

\section{Assessment of Headache and Headache Classification}

Subjects who responded positively to the question "Did you have any headache during the past 7 days/3 months/ 6 months?" were classified as headache sufferers. They answered further questions regarding their headache characteristics such as symptoms, duration, frequency, and intensity to identify different types of headache according to the criteria of the German translation of the International Classification of Headache Disorders, 2nd edition (ICHD-II). ${ }^{13}$ Headache was classified as migraine (including the subtypes migraine with or without aura, chronic migraine, probable migraine, and probable chronic migraine), TTH (including the subtypes infrequent episodic TTH, frequent episodic TTH, chronic TTH, probable infrequent episodic TTH, probable frequent episodic TTH, and probable chronic TTH), a combination of migraine plus TTH, or miscellaneous headache. Details are described elsewhere. ${ }^{3-5}$ In our present analysis, we distinguished between migraine and TTH. Patients suffering migraine plus TTH were also classified as migraine patients.

\section{Assessment of Lifestyle Factors, Stress, Neck Tension, and Socioeconomic Variables}

Previously, the impact of lifestyle factors (coffee consumption, physical inactivity, alcohol consumption, and smoking), 4 stress, ${ }^{3}$ and muscle pain in the head, neck, and shoulder region $^{5}$ on headache was described, classifying the exposure on an ordinal scale. Because PARF calculation with our approaches requires binary classification of the risk factors, we dichotomized our variables as follows: Adolescents were classified to drink alcohol (alcoholic mixed drinks, cocktails, liquor) if they answered the question "How much alcohol (i.e., cocktails, mixed drinks and hard liquor) do you normally drink?" with any answer that included drinking alcohol (i.e., $<1$ glass per moth, $<1$ glass per week, 1 and more glasses per week). Analogously, adolescents were considered to drink coffee if they answered the question "How much coffee do you drink?" with any answer that included drinking coffee (i.e., $<1$ cup per week, $<1$ cup per day, 1 and more cups per day). Smoking was assessed by the question "Do you smoke?" Subjects who answered with "sometimes" or "daily" were classified as smokers. Those who answered "never" were classified as nonsmokers. Physical inactivity was determined by the question "How often do you do sports during leisure time?" Adolescents were considered to be physically inactive if they answered "none at all" or "less than once a month." Otherwise (i.e., more than once a month), they were classified as physically active. The presence of muscle pain in the head, neck, and shoulder region was assessed by the question "Do you suffer from muscle pain in shoulder, neck or head?," which could be answered with yes or no.

The questionnaire further included questions regarding stressful experience. Theses were constructed according to the Trier Inventory of Chronic Stress, a 57-item self-report instrument yielding nine dimensions extracted by factor analysis. $^{14}$ One of these dimensions is the chronic stress 
screening scale, consisting of 12 items taken from the other dimensions. This is suggested to be used as a global measure for chronic stress experience.

Socioeconomic variables were assessed and categorized in the following way. Monthly amount of adolescents' pocket money was dichotomized " 50 Euro or less" versus "more than 50 Euro.” Students' own income was considered "any” versus "nothing." Questions on parents' employment status were taken from the German Health Interview and Examination Survey for Children and Adolescents and considered "fulltime" or "not full-time."

\section{Statistical Analysis}

The association between lifestyle factors and headache and headache subtypes was assessed with logistic regression models adjusted for sex, age, and socioeconomic variables (pocket money, students' own income, parents' employment status) as potential confounding variables (Model 1) and additionally with mutual adjustment (Model 2). Odds ratios with $95 \%$ confidence intervals were estimated for these two models.

To assess the potential impact of the identified risk factors on a population level, PARFs were estimated. This estimate considers not only the strength of the association but additionally the prevalence of exposure in the investigated population. One of the most frequently applied approaches calculating the PARF is the Levin formula (Approach 1):

$$
\mathrm{PARF}_{\text {Levin }}=\frac{P \cdot(R R-1)}{1+P \cdot(R R-1)}
$$

where $P$ denotes the prevalence of the risk factor and $R R$ stands for the relative risk estimate (in cross-sectional studies approximated by the odds ratio). ${ }^{6}$ This approach has a couple of disadvantages ${ }^{8}$ : It overestimates the preventive potential, because often the total sum of the PARFs of the considered risk factors exceeds $100 \%$. Even if adjusted odds ratio estimates from multivariable logistic regression are entered into this formula, the PARF is likely to be a biased estimator, as noted by Greenland. 7,15

Among several alternative approaches discussed in the literature, ${ }^{8,9,16}$ model-based approaches like the maximum likelihood estimator of attributable fraction are considered to be more robust according to Benichou ${ }^{8}$ and Greenland and Drescherand. ${ }^{10}$ For this approach the formula for the PARF is given by

$$
\operatorname{PARF}=\frac{N_{\mathrm{o}}-N_{\mathrm{e}}}{N_{\mathrm{o}}}
$$

where $N_{\mathrm{o}}$ denotes the observed number of adolescents with headache and $N_{\mathrm{e}}$ is the expected number of adolescents with headache after removal of the exposure to the risk factor. To estimate $N_{\mathrm{e}}$ one removes the risk factor of interest from the model by simply setting this covariate at zero for all individuals. Summing up the predicted probabilities for each individual gives the expected number of adolescents with headache after removal of the exposure. To get a more precise estimate, the sequential removal of risk factors is taken into account as previously proposed. ${ }^{11}$ To avoid the dependence of the order of the risk factors removal from the model, the average of all possible orders is taken. This approach is presented by Eide and Gefeller and is called the average attributable fraction (Approach 2). ${ }^{11}$ We used codes for the software packages SAS provided by Rückinger et al to calculate the PARF with this method. ${ }^{16}$

To analyze the variation of the PARF by age, we divided the study population into three age groups: 317 students younger than 16 years ( 14 to 15 years), 555 students age 16 years, and 388 students aged 17 years and older (17 to 20 years). Using the SAS codes for Approach 2, it is not possible to estimate PARFs for different age groups while assuming fixed effects for the whole population. Therefore, PARFs for the age groups were calculated using Levin formula only. We tested variation by age using the Cochrane-Armitage test for trends. Based on the PARF values for each age group, we calculated the expected number of cases if the risk factor was absent in the population and applied the test to each risk factor. Values of $p<0.05$ were considered to indicate a significant trend.

\section{Results}

\section{Prevalence of Headache}

Some $83.1 \%$ ( $n=1,047)$ of all students reported headache at least once during the past 6 months. The most frequent type of headache was pure TTH $(48.7 \%, n=614)$, whereas $10.2 \%$ of subjects $(n=120)$ suffered from pure migraine. Combined migraine plus TTH was reported by $19.8 \%$ of students ( $n=249)$. As for the remaining $4.4 \%$ of participants $(n=55)$, the type of headache could not be classified according to the ICHD-II criteria and so were considered to have miscellaneous headache.

\section{Prevalence of Risk Factors}

- Table 1 shows the prevalence of the risk factors for adolescents with any headache in descending order: Alcohol consumption was most prevalent; followed by coffee consumption; muscle pain in the head, neck, and shoulder region; smoking; physical inactivity; and chronic stress. Regarding the different headache types, muscle pain in the head, neck, and shoulder region and chronic stress appear considerably more common in the migraine group. The other risk factors show only slightly different prevalences between the migraine and TTH group.

\section{Association between Selected Lifestyle Factors; Muscle Pain in the Head, Neck, and Shoulder Region; Stress; and Headache}

The strongest association in Model 1 for any headache could be seen for chronic stress and muscle pain in the head, neck, and shoulder region (-Table 1, second column). Model 2 (with mutual adjustment) shows slightly modified effects for any headache (-Table 1, third column): The impact of coffee consumption, physical inactivity, and smoking changed only marginally but were no longer significant, whereas the effects of all other risk factors decreased considerably. Applying 


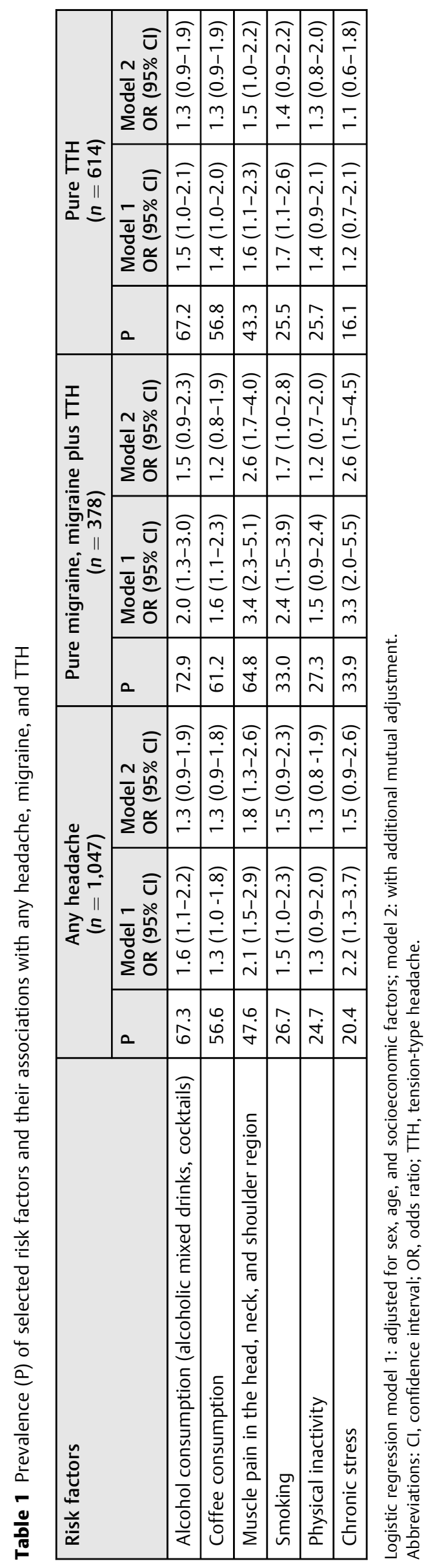

Model 2, the strongest association was seen for muscle pain in the head, neck, and shoulder region, followed by chronic stress and smoking showing nearly the same impact, respectively. Regarding migraine and TTH separately, considerable differences of the effect sizes for muscle pain in the head, neck, and shoulder region and chronic stress could be observed using both Model 1 and Model 2 (-Table 1). All other risk factors vary only little between the groups, which is summarized in -Table 1. Using Model 2 smoking shows significant effects for migraine but not for TTH. Physical inactivity missed significance in both group for both models.

\section{Population-Attributable Risk}

Both approaches consistently yielded muscle pain in the head, neck, and shoulder region to be the strongest populationattributable risk factor for any headache followed by alcohol consumption. The lowest PARF was observed for physical inactivity (-Table 2, first two columns). For the other risk factors, results depend on the applied approach: Levin formula (Approach 1) estimated a higher PARF for chronic stress, whereas the model-based approach (Approach 2) yielded stronger population-attributable effects for coffee consumption. The total risk explained amounts up to $79.9 \%$ using Approach 1. Using Approach 2, which seems to be more appropriate, about $19.7 \%$ of the risk of headache in the population is explained.

The overall impact on migraine for removal of all six risk factors amounts to $43.8 \%$ (Approach 2) and is considerably higher than the overall impact of the risk factors on TTH. The calculations with Approach 1 confirmed these results. These differences are mainly explained by the high PARF values for muscle pain in the head, neck, and shoulder region and chronic stress in the migraine group. The PARF value of muscle pain for migraine amounts to more than twice as much as for TTH. For chronic stress the PARF for migraine is even 20 times higher than for TTH. All other PARF values differ only slightly, as shown in - Table 2.

\section{Age Dependency of the Population-Attributable Risk}

An increase of prevalence was observed for nearly all risk factors (except for coffee consumption and muscle pain in the head, neck, and shoulder region) by the age of 17 and older compared with the youngest students (-Fig. 1). The prevalence for consumption of alcohol showed the highest increase (from 54.7 to $74.8 \%$ ), followed by smoking and physical inactivity (from 22.6 to $32.9 \%$ and 19.4 to $29.9 \%$, respectively). Thus, also the impact of these risk factors increased with age as provided in - Fig. 1 (Approach 1). The PARFs of alcohol consumption (from 15.3 to $19.8 \% ; p=0.034$ ), smoking (from 9.2 to $12.8 \%, p=0.025$ ), and physical inactivity (from 4.8 to $7.2 \% ; p=0.033$ ) changed even significantly. A slight rise could be observed for chronic stress, marginally missing the significance level of $5 \%(p=0.0534)$, and coffee consumption ( $p=0.178)$, whereas muscle pain in the head, neck, and shoulder region ( $p=0.208$ ) seems to appear to be independent of age. Finally, the total risk in students aged 17 years and older is $18.9 \%$ higher than the total risk in students younger than 16 years (set as $100 \%$ ). 
Table 2 PARF based on two different approaches (Levin formula, Appr. 1, and the average attributable fraction, Appr. 2) estimated for any headache, migraine, and TTH

\begin{tabular}{|c|c|c|c|c|c|c|}
\hline \multirow[t]{2}{*}{ Risk factors } & \multicolumn{2}{|c|}{$\begin{array}{l}\text { Any headache } \\
(n=1,047)\end{array}$} & \multicolumn{2}{|c|}{$\begin{array}{l}\text { Pure migraine, } \\
\text { migraine plus TTH } \\
(n=378)\end{array}$} & \multicolumn{2}{|c|}{ Pure TTH $(n=614)$} \\
\hline & $\begin{array}{l}\text { PARF } \\
\text { (Appr. 1) }\end{array}$ & $\begin{array}{l}\text { PARF } \\
\text { (Appr. 2) }\end{array}$ & $\begin{array}{l}\text { PARF } \\
\text { (Appr. 1) }\end{array}$ & $\begin{array}{l}\text { PARF } \\
\text { (Appr. 2) }\end{array}$ & $\begin{array}{l}\text { PARF } \\
\text { (Appr. 1) }\end{array}$ & $\begin{array}{l}\text { PARF } \\
\text { (Appr. 2) }\end{array}$ \\
\hline $\begin{array}{l}\text { Alcohol consumption (alcoholic mixed drinks, } \\
\text { cocktails) }\end{array}$ & 18.0 & 5.1 & 24.4 & 8.9 & 15.1 & 5.2 \\
\hline Coffee consumption & 9.5 & 3.5 & 10.7 & 4.1 & 14.2 & 3.8 \\
\hline Muscle pain in the head, neck, and shoulder region & 27.9 & 5.9 & 43.8 & 16.6 & 18.7 & 4.2 \\
\hline Smoking & 9.0 & 2.2 & 15.3 & 5.0 & 9.4 & 2.2 \\
\hline Physical inactivity & 4.1 & 1.1 & 4.4 & 1.1 & 6.1 & 1.4 \\
\hline Chronic stress & 11.4 & 1.9 & 24.6 & 8.0 & 1.2 & 0.3 \\
\hline Total & 79.9 & 19.7 & 123.2 & 43.8 & 64.6 & 17.1 \\
\hline
\end{tabular}

Abbreviations: Appr, approach; PARF, population-attributable risk fraction; TTH, tension-type headache.

\section{Discussion}

Although the impact of identified risk factors was of a relevant size on the individual level, with odds ratios of 1.3 to 1.8 , the impact of all risk factors combined in the population accounted for only about $20 \%$ of the population risk for headache in our calculation. For migraine the overall impact was considerably higher and amounted to over $40 \%$.

To assess whether the impact of risk factors for headache on the population level has been studied previously, we searched MEDLINE with the search terms "headache AND (population attributable fraction OR population attributable risk OR PARF)." We could not identify any matching results. Therefore, we believe our study is one of the first to calculate PARFs for headache in adolescents. Such an approach has previously been more often used in the literature concerning genetics to estimate the relative influence of environment and genes on headache. ${ }^{17}$
The lack of publications of the population-based impact of risk factor for headache may reflect uncertainties about the appropriate method for estimation. As previously mentioned, our estimations show some limitations as well. All approaches depend strongly on the logistic regression model. Because not all risk factors and confounders are really known and others, for example adverse family relations, are difficult to quantify, we had to restrict the analysis to only some of them. Given the fact that well-established risk factors for headache such as genetic disposition, style of coping with headache, and so on are not considered, it can be expected that our model explains considerably less than $100 \%$ of the population-based risk of headache. Therefore, a total PARF of nearly $80 \%$ calculated with the more traditional Levin formula seems quite unlikely high. We presented estimates based on this approach, however, because this is the classical method to calculate PARFs and to assess their age dependency.

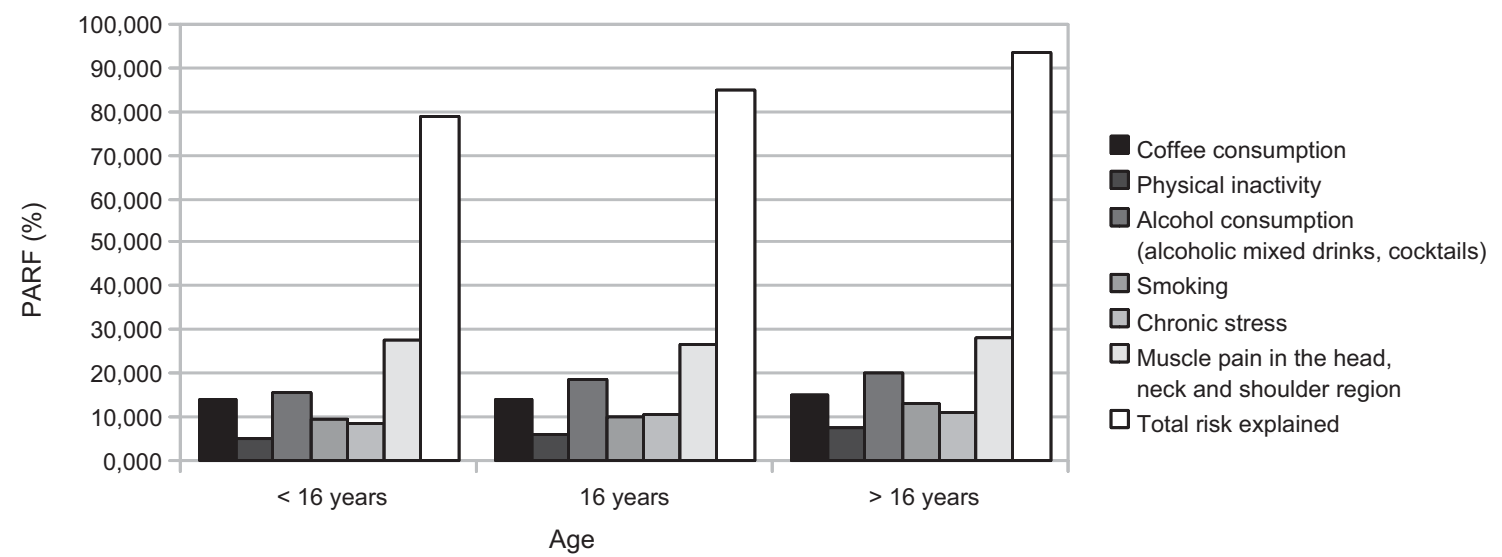

Fig. 1 Variation of population-attributable risk fraction (PARF) by age. Approach 1: Levin formula. 
Unlike the standard approach (Levin formula), modelbased approaches are considered more robust and less prone to overestimation, as previously shown. ${ }^{16}$ Thus, we decided also to apply another approach for estimating PARFs. The sequential removal as used in Approach 2 is a further elaboration and yields the most probable results. The estimated attributable risk of the risk factors considered with this approach was much lower (about 20\%).

In conclusion, we provide the first evaluation of PARF related to risk factors for headache in adolescents, which might be amenable to interventions. Knowledge of PARF is a prerequisite for the conception of prevention concerning headache in the pediatric population: Up to $20 \%$ of the headaches in adolescents might be prevented by appropriate interventions, with increasing proportions by age due to increasing consumption of alcohol, smoking, and physical inactivity by age. Interventions should particularly aim to reduce consumption of alcohol and coffee and muscle pain. Migraine patients are more likely to benefit from interventions, with most effects attainable by reduction of chronic stress and muscle pain in the head, neck, and shoulder region because the attributable risk for migraine was surprisingly higher than for TTH. The identification of muscle pain as a main risk factor for migraine as opposed to TTH could support the theory of the cervicotrigeminale loop interacting between the brainstem and C-1 supplied muscles in cases of migraine. Insufficient habituation as demonstrated for migraine could play a key role in the relation between peripheral muscle pain and central regulation of pain. As well, the difference might be because the most important risk factors for TTH were not identified in our study or because genetic impact is higher on TTH than previously assumed. The rising proportion of potentially preventable cases by age points to the need to extend these analyses to adults to assess whether the increase of the PARFs stops and decreases at a certain age in adulthood and whether the main risk factors for headache might change by age as suggested by results of the German Migraine and Headache Society Headache study. ${ }^{12}$

\section{References}

1 Ellert U, Neuhauser H, Roth-Isigkeit A. [Pain in children and adolescents in Germany: the prevalence and usage of medical services. Results of the German Health Interview and Examination Survey for Children and Adolescents (KiGGS)]. Bundesgesund Gesundheitsforsch Gesundh 2007;50(5-6):711-717

2 Stovner LS, Andree C. Prevalence of headache in Europe: a review for the Eurolight project. J Headache Pain 2010;11:289-299

3 Milde-Busch A, Blaschek A, Heinen F, et al. Associations between stress and migraine and tension-type headache: results from a school-based study in adolescents from grammar schools in Germany. Cephalalgia 2011;31(7):774-785

4 Milde-Busch A, Blaschek A, Borggräfe I, Heinen F, Straube A, von Kries R. Associations of diet and lifestyle with headache in highschool students: results from a cross-sectional study. Headache 2010;50(7):1104-1114

5 Blaschek A, Milde-Busch A, Straube A, et al. Self-reported muscle pain in adolescents with migraine and tension-type headache. Cephalalgia 2012;32(3):241-249

6 Levin ML. The occurrence of lung cancer i... [Acta Unio Int Contra Cancrum. 1953] - PubMed - NCBI [Internet]. Available from http://www.ncbi.nlm.nih.gov/pubmed?term=(Levin\%5BAuthor\%5D)\%20AND\%20The\%20occurence\%20of\%20lung\%20cancer\% $5 \mathrm{BTitle} \% 5 \mathrm{D}$

7 Greenland S. Morgenstern corrects a conceptual error. Am J Public Health 1983;73(6):703-704

8 Benichou J. A review of adjusted estimators of attributable risk. Stat Methods Med Res 2001;10(3):195-216

9 Gefeller O. Comparison of adjusted attributable risk estimators. Stat Med 1992;11(16):2083-2091

10 Greenland S, Drescher K. Maximum likelihood estimation of the attributable fraction from logistic models. Biometrics 1993;49 (3):865-872

11 Eide GE, Gefeller O. Sequential and average attributable fractions as aids in the selection of preventive strategies. J Clin Epidemiol 1995;48(5):645-655

12 Winter AC, Hoffmann W, Meisinger C, et al. Association between lifestyle factors and headache. J Headache Pain 2011;12(2): 147-155

13 Headache Classification Subcommmittee of the International Headache Society. The International Classification of Headache Disorders, 2nd edition. Cephalalgia 2004;(40):669-74

14 Schulz P. Trier Inventory of Chronic Stress. Goettingen: Hogrefe; 2004

15 Morgenstern H. Uses of ecologic analysis in epidemiologic research. Am J Public Health 1982;72(12):1336-1344

16 Rückinger S, von Kries R, Toschke AM. An illustration of and programs estimating attributable fractions in large scale surveys considering multiple risk factors. BMC Med Res Methodol 2009;9:7

17 Ulrich V, Gervil M, Olesen J. The relative influence of environment and genes in episodic tension-type headache. Neurology 2004; 62(11):2065-2069 